\title{
Prophages mediate defense against phage infection through diverse mechanisms
}

\author{
Joseph Bondy-Denomy ${ }^{1,7}$, Jason Qian ${ }^{1}$, Edze R Westra ${ }^{2}$, Angus Buckling², \\ David S Guttman $^{3,4}$, Alan R Davidson ${ }^{1,5}$ and Karen L Maxwell ${ }^{6}$ \\ ${ }^{1}$ Department of Molecular Genetics, University of Toronto, Toronto, ON, Canada; ${ }^{2}$ Environment and \\ Sustainability Institute, Biosciences, University of Exeter, Penryn, UK; ${ }^{3}$ Centre for the Analysis of Genome \\ Evolution and Function, University of Toronto, Toronto, ON, Canada; ${ }^{4}$ Department of Cell and Systems \\ Biology, University of Toronto, Toronto, ON, Canada; ${ }^{5}$ Department of Biochemistry, University of Toronto, \\ Toronto, ON, Canada and ${ }^{6}$ Donnelly Centre for Cellular and Biomolecular Research, University of Toronto, \\ Toronto, ON, Canada
}

\begin{abstract}
The activity of bacteriophages poses a major threat to bacterial survival. Upon infection, a temperate phage can either kill the host cell or be maintained as a prophage. In this state, the bacteria carrying the prophage is at risk of superinfection, where another phage injects its genetic material and competes for host cell resources. To avoid this, many phages have evolved mechanisms that alter the bacteria and make it resistant to phage superinfection. The mechanisms underlying these phentoypic conversions and the fitness consequences for the host are poorly understood, and systematic studies of superinfection exclusion mechanisms are lacking. In this study, we examined a wide range of Pseudomonas aeruginosa phages and found that they mediate superinfection exclusion through a variety of mechanisms, some of which affected the type IV pilus and O-antigen, and others that functioned inside the cell. The strongest resistance mechanism was a surface modification that we showed is cost-free for the bacterial host in a natural soil environment and in a Caenorhabditis. elegans infection model. This study represents the first systematic approach to address how a population of prophages influences phage resistance and bacterial behavior in $\boldsymbol{P}$. aeruginosa. The ISME Journal (2016) 10, 2854-2866; doi:10.1038/ismej.2016.79; published online 3 June 2016
\end{abstract}

\section{Introduction}

Bacteriophages (phages) are viruses that infect bacteria. They are the most abundant biological entity on earth, and with an estimated global population of $10^{31}$, they outnumber bacteria by a factor of 10 (Bergh et al., 1989; Wommack and Colwell, 2000). Phages are tremendously diverse and are found in every habitat that has been colonized by bacteria, including water, soil and gastrointestinal ecosystems. Their predation of bacteria has a strong influence on microbial populations, affecting the performance of food webs, nutrient cycling, biodiversity, species distribution and genetic transfer (Suttle, 2005, 2007).

Phages are classified as lytic or temperate, depending on their lifestyle. A successful infection by lytic phages always leads to cell lysis, whereas infection

Correspondence: K Maxwell, Donnelly Centre for Cellular and Biomolecular Research, University of Toronto, Toronto, ON, Canada M5S3E1.

E-mail: karen.maxwell@utoronto.ca

${ }^{7}$ Current address: Department of Microbiology and Immunology, University of California San Francisco, San Francisco, CA, USA. Received 6 January 2016; revised 31 March 2016; accepted 4 April 2016; published online 3 June 2016 by a temperate phage can lead to lysis or lysogeny. During lysogeny, the bacterial host and phage fitness interests are aligned in a symbiotic relationship. It is therefore advantageous for the prophage to express genes that increase fitness of the host cell by a process known as lysogenic conversion. Indeed, phages have been shown to encode genes that confer phage resistance through a variety of mechanisms (Samson et al., 2013). For example, superinfection exclusion proteins expressed from prophages that inhibit further phage infection have been characterized in Escherichia coli phages HK97 (Cumby et al., 2012), $\phi 80$ (Uc-Mass et al., 2004), Salmonella phage P22 (Susskind et al., 1974) and Streptococcus phage TP-J34 (Sun et al., 2006). The mechanisms by which these proteins block further phage infection include interacting with the cytoplasmic membrane and blocking phage genome injection, and interacting with the phage receptor on the bacterial outer membrane and blocking phage binding. In Pseudomonas phage D3112, the Tip protein inhibits bacterial twitching motility through interaction with the type IV pilus (T4P) assembly ATPase and protects against infection by phage MP22 (Chung et al., 2014). As multiple Pseudomonas aeruginosa phages rely on the T4P for infection (Bradley, 1972, 1973), 
this was proposed as a general mechanism for blocking further phage infection. Although there are many individual examples of prophages that express genes that prevent superinfection, it is unclear both how widespread each of these different superinfection resistance mechanisms are and what fitness consequences they have for their hosts.

In this study we use $P$. aeruginosa, an organism that naturally possesses an abundant and diverse population of prophages (Winstanley et al., 2009), to characterize the prevalence of prophage-mediated superinfection exclusion and determine the mechanisms that underlie it. We generated an unbiased collection of lysogens so that we could undertake a systematic examination of range and fitness costs of superinfection exclusion mechanisms encoded by phages for a single bacterial host. Our results highlight the variable phenotypes that a related group of prophages can impart on a bacterial host.

\section{Results}

Establishment of a collection of P. aeruginosa strain PA14 lysogens

To interrogate systematically the effects of prophages on the physiology of $P$. aeruginosa, we generated a collection of temperate phages by using mitomycin $\mathrm{C}$ to induce the prophages present in a set of 88 clinical and environmental isolates of $P$. aeruginosa (Supplementary Table S1). These strains were selected for maximal diversity as assessed by point of geographic isolation and mutilocus sequence typing (Supplementary Figure S1), a commonly used method for assessing strain diversity (Parkins et al., 2014). Serial dilutions of the induced lysates were plated on lawns of $20 \mathrm{P}$. aeruginosa indicator strains selected from the collection of 88, including the laboratory strains PA14, PAO1 and PAK, to provide a broad host range and maximize the number of plaques that could be detected. We found that $56 / 88(66 \%)$ of the strains produced at least one phage that could form plaques on this set of strains, with two strains producing three phages, and seven producing two phages. Strain PA14 was determined to be the most permissive strain for phage replication, with 28 of the isolated phages able to form plaques on it. Using these 28 newly isolated phages and two previously characterized phages, MP22 and MP29 (Chung and Cho, 2012), we created 30 different lysogenic strains of PA14, each containing one of the 30 phages integrated as a prophage. The identities of the individual lysogens were confirmed by growing the strains overnight and performing plaque assays using phages spontaneously produced by these cultures. As was previously observed with $P$. aeruginosa prophages (James et al., 2012), the spontaneous induction frequencies were quite high, with overnight cultures containing between $10^{5}$ and $10^{9}$ plaque-forming units per $\mathrm{ml}(\mathrm{PFU}$ per $\mathrm{ml}$ ) in the absence of exogenous chemical or ultraviolet induction (Supplementary Figure S3).
Lysogens display a variety of phage resistance phenotypes

Prophages can mediate resistance to subsequent phage infection through repressor-based immunity and potentially other mechanisms (Salmon et al., 2000). To assess the range of phage resistance mediated by our collection of lysogens, the ability of each individual lysogen to resist the plaquing of the 30 PA14-infecting phages was measured, as well as mutants lacking the T4P and $\mathrm{O}$-antigen, which are commonly used as phage receptors (Figure 1). As seen in Figure 1, 28 of 30 lysogens were resistant ( $>10^{3}$-fold reduction in plaquing efficiency, denoted by dark blue squares) to at least one other phage besides the phage from which the lysogen was made. Most lysogens also mediated 'partial resistance' to some phages (denoted by cyan squares) meaning that plaquing activity was decreased 10 - to 100 -fold. The patterns of resistance displayed by the lysogens were diverse, with 12 distinct patterns observed. The resistance pattern of a lysogen was defined as 'distinct' if its resistance to at least three phages differed from that of any other lysogen that was not in the same group.

The 12 distinct resistance patterns were classified into five broad groups (H, M1, M2, L1 and L2) based on the strength of their resistance and the range of phages that could plaque on them. Lysogens in the high resistance $(\mathrm{H})$ group decreased the plaquing efficiency of at least 19 phages by $>10^{3}$-fold. There were two medium resistance groups showing $>10^{3}$ fold resistance between 7 and 11 phages (group M1) and 6 phages (group M2). The two low resistance groups were L1, which were resistant to only two or three phages, and L2, which were completely resistant only to the phage used to create the lysogen. Although the resistance patterns within the $\mathrm{H}$ and M1 groups were broadly similar, four distinct patterns were discernible in each of these groups, and two distinct patterns were seen in the L2 group. In summary, the resistance patterns of this group of prophages exhibited high variability, with some prophages showing broad superinfection exclusion, and others unable to resist superinfection by any other phages. This suggests high diversity in the mechanisms that underlie superinfection exclusion based resistance in this group of phages that share the same host.

Many bacterial genomes harbor multiple prophages (Canchaya et al., 2003). We therefore investigated if the presence of multiple prophages conferred a broader range of superinfection exclusion as a result of an increased diversity of exclusion mechanisms. A PA14 double lysogen containing both an M2 (JBD44) and $\mathrm{H}$ (MP29) prophage displayed complete resistance to all phages but JBD24. A triple lysogen was made, PA14 (JBD44/MP29/JBD24), and this strain was resistant to every phage in our collection (Figure 1, '2x' and ' $3 x$ ' columns). To ensure that the extensive resistance displayed by our lysogens was not an artifact of our strain collection or our method of isolating phages, 


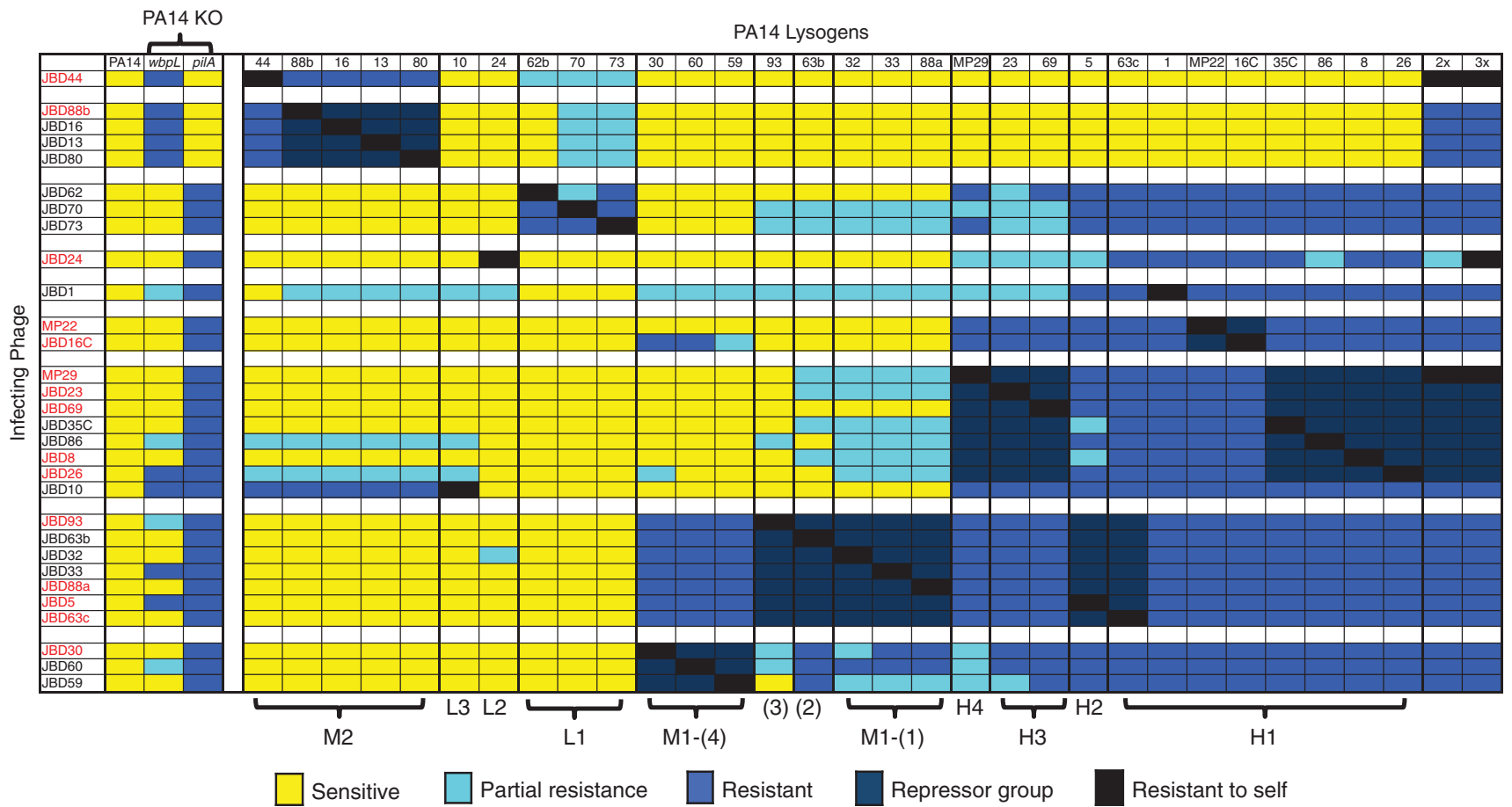

Figure 1 Phage sensitivity profiles of strains in the PA14 prophage collection. Wild-type PA14, mutant strains lacking the O-antigen $(w b p L)$ or pilus (pilA), and PA14 lysogenized with 30 different phages (x axis) were infected with a group of 30 phages (y axis). The outcome of the infection is shown in the color scale in the legend. Double (2x, MP29 and JBD44a) and triple (3x, MP29, JBD44a and JBD24) lysogens were made to assess the additivity of resistance. Phage groupings along the y axis represent homoimmune repressor families, and repressor-mediated resistance is shown in navy blue. Sequenced phage genomes are highlighted in red.

we assessed the plaquing activity of three previously isolated siphophages DMS3 (Zegans et al., 2009), MP38 (Heo et al., 2007) and D3112 (Wang et al., 2004), and four myophages from the Lindberg typing set (Ackermann et al., 1988). These phages were also unable to form plaques on many of the lysogens, with five of the six phages tested being unable to plaque on at least 8 of the 17 strains tested (Supplementary Figure S4). Phage 1214 provides one counterexample to this trend, as it was able to plaque well on all lysogens except the M2 group. Intriguingly, this was the only phage able to plaque on mutants lacking either the $\mathrm{T} 4 \mathrm{P}$ or the O-antigen. Therefore, the resistance mechanisms observed here are additive and not unique to the specific set of phages isolated in this work.

\section{Phages display a variety of lysogen infectivity phenotypes}

From the above experiment, it is clear that phages can carry a wide range of superinfection resistance mechanisms. However, it is less clear if and how this correlates with phage infectivity on the lysogenic hosts. We therefore analyzed the host ranges for the phages when infecting the various lysogen groups as defined above. In many cases, phages belonging to the same lysogen groups displayed similar infectivity ranges, and were typically unable to infect members of the same lysogen group. For example, the M2 group of phages were all unable to plaque on lysogens of any member of the M2 group, but could form plaques robustly on most other lysogens (Figures 1 and 2). This group of phages was distinguished from all of the other phages by their ability to form plaques on a PA14 strain lacking its T4P (pilA mutant; Figure 1), whereas being unable to form plaques on strains lacking the O-antigen ( $w b p L$ mutant) of the surface lipopolysaccharide. Among the phages requiring the T4P, those within the M1 group displayed almost identical patterns of plaquing, propagating only on the L and M2 groups of lysogens. The phages within other lysogen groups displayed more highly variable plaquing abilities. For example, phage JBD1 showed partial plaquing on numerous lysogens (Figure 2 and Supplementary Figure S5), whereas JBD5 (from the same resistance group, H1) was unable to plaque on these same lysogens. Interestingly, the L2 group phage, JBD24, which as a lysogen was resistant to only one phage beyond itself, was able to form plaques on more lysogens than the other T4P-requiring phages (Figures 1 and 2 and Supplementary Figure S5). By contrast, the other L2 group phage, JBD10, was completely unable to infect all $\mathrm{H}$ group lysogens and was also the only T4P-requiring phage to be completely inhibited by the M2 prophages.

\section{Closely related phage genomes display localized} gene diversity

To gain insight into the genetic backgrounds leading to the prophage-mediated bacterial phenotypes, we 


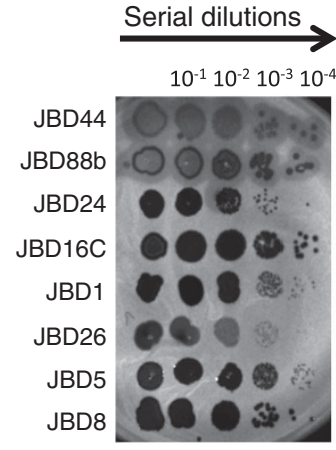

PA14

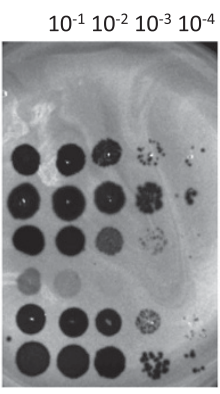

PA14(JBD44a)

M2

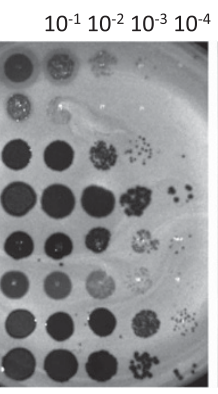

PA14(JBD73)

L2
$10^{-1} 10^{-2} 10^{-3} 10^{-4}$

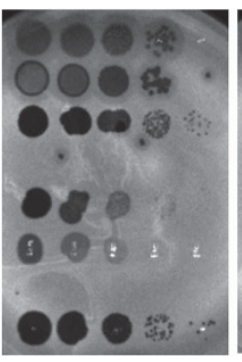

PA14(JBD30)

M1-4
$10^{-1} 10^{-2} 10^{-3} 10^{-4}$

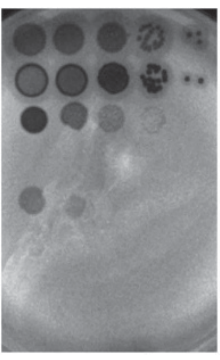

PA14(JBD23) $\mathrm{H} 1-3$
$10^{-1} 10^{-2} 10^{-3} 10^{-4}$

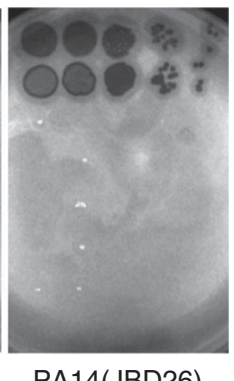

$\mathrm{H} 1-1$

Figure 2 Phage plaque formation assays. Eight different phage lysates were applied in 10-fold serial dilutions to lawns of wild-type PA14, or the indicated PA14 lysogens and plaque formation was assessed. The resistance group that a given lysogen belongs to (see Figure 1) is shown. See Supplementary Figure S5 for more plaque assays.

sequenced the genomes of 12 phages used to make our PA14 lysogen collection (Supplementary Table S2). We found that each of these phages belong to the Siphoviridae family, which possess a doublestranded DNA genome packed inside an icosahedral head attached to a long, non-contractile tail (see Supplementary Figure S6 for electron microscopy images). Two other phages sequenced in this study (JBD44 and JBD88b) were siphophages (Supplementary Figure S6) that required the O-antigen for infection, and were found to be similar to each other in gene order and sequence identity (see Supplementary Table S2 for genome information). These phages possess integrase genes and they integrate as prophages at specific sites in the bacterial genome (Supplementary Figure S7a). The genome arrangements of these phages are similar to $P$. aeruginosa phages D3 and phi297 (Kropinski, 2000; Bourkal'tseva et al., 2011), and $65 \%$ of the proteins encoded by these phages are clearly related (BLAST $e$-value $<0.001$ ) to proteins encoded by one or the other of these phages (Supplementary Table S2). The other 10 phages were unrelated to JBD44 and JBD88b, but were closely related to each other. Their genomes contained Mu-like transposase genes, which are required for replication of the phage genome and the random insertion of the phage genome into the host chromosome upon lysogen formation. Random integration was confirmed for JBD26 via Southern blot (Supplementary Figure S7b). These phages all required the T4P for infection, and displayed high sequence similarity with previously characterized phages MP22 and MP29, which were also included in our lysogen analysis (Figure 1). As 10 of 12 phages that we isolated had very closely related genome sequences, we wondered if there was a bias introduced by the initial selection of phages that could form plaques on PA14. Thus, we chose five additional phages that were unable to infect strain PA14 from our collection of 70 temperate Pseudomonas phages and sequenced their genomes. We found that these phages were members of three different phage families unrelated to the two groups described above. Phages JBD25, JBD18 and JBD67 were all similar to Mu-like $P$. aeruginosa siphophage B3, JBD68 was similar to $P$. aeruginosa siphophage F10 and JBD90 was similar to $P$. aeruginosa podophage F116 (Supplementary Table S2). Thus, it appears that selection for growth in the PA14 host strain background did bias our phage collection, and illustrates the importance of using many indicator strains when assessing the diversity of phage populations.

To determine if there was a correlation between the gene complement of different phages and the resistance displayed by their corresponding lysogens, we compared the genomes of the 10 MP22-like phages. The average number of open reading frames encoded in these genomes, including phages MP22 and MP29, was $57 \pm 3$, and $\sim 80 \%$ of these open reading frames encoded proteins that were conserved among all phages, with pairwise amino-acid sequence identities of $80-90 \%$ in most cases (Supplementary Tables S3 and S4). These genes were arranged in a conserved order, and the predicted functions of 30 of the 44 conserved genes could be annotated (Figure 3 and Supplementary Table S3). In addition to the highly conserved genes, $\sim 20 \%$ of the genes in each of these genomes were not found in every genome. We identified a total of 32 families of non-conserved or 'accessory' genes among the 12 MP22-like genomes. Members of these accessory gene families were often found in many of these MP22-like genomes, but no genome had exactly the same complement of accessory genes (Supplementary Table S3). Aside from the previously characterized Tip (Chung et al., 2014) and anti-CRISPR genes (Bondy-Denomy et al., 2013; Pawluk et al., 2014), the accessory genes encode proteins of unknown function. Homologs of $50 \%$ of these accessory genes were found only among phages closely related to the MP22-like phages described here. Homologs of the other accessory genes were found in one or more different type of 


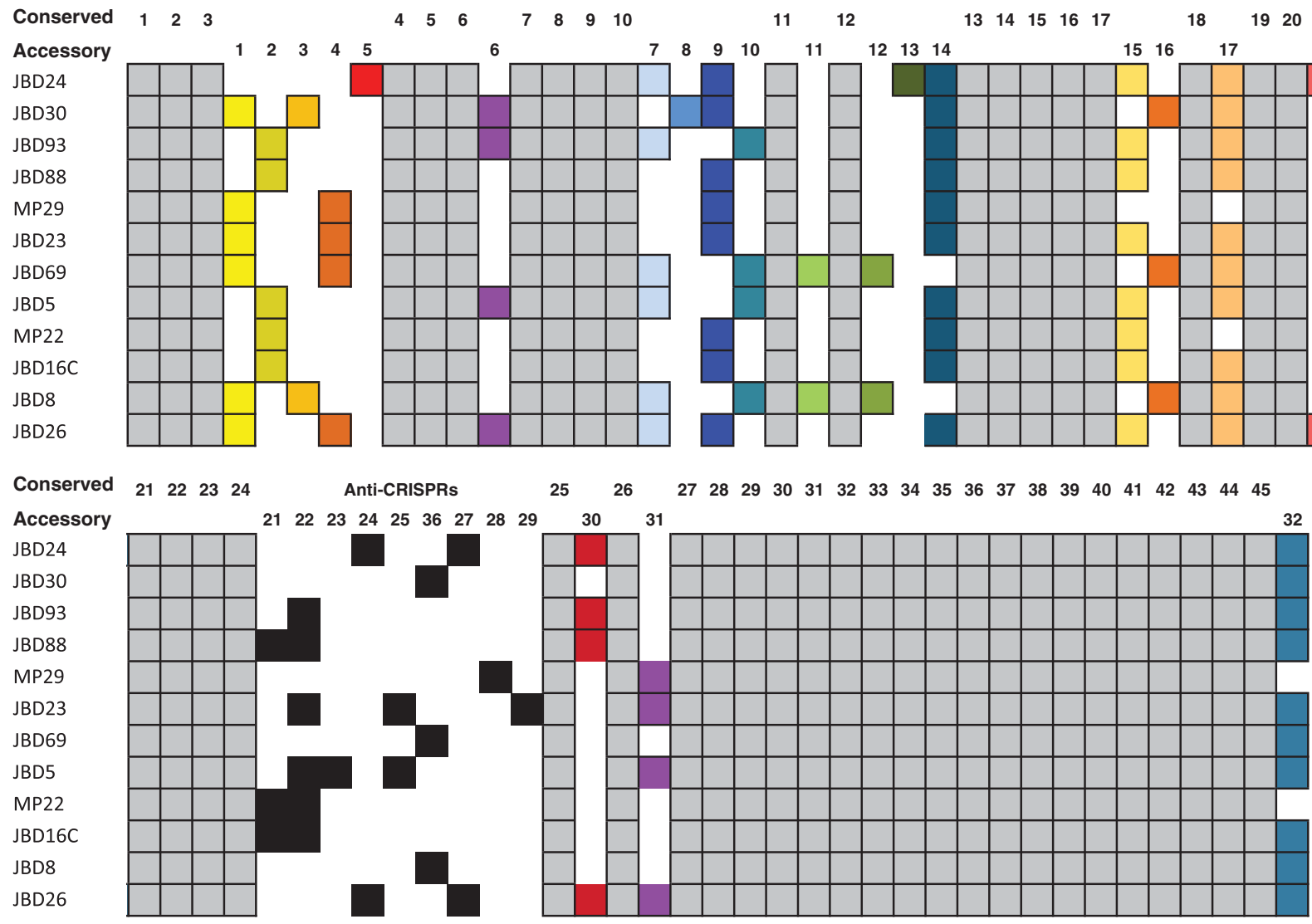

Figure 3 Alignment of the phage genomes highlights the presence of a highly variable accessory genome. Each box represents a single open reading frame, with gray boxes denoting conserved genes, and colored boxes denoting non-conserved 'accessory genes'. Different vertical positions and colors for the accessory genes indicate that they encode proteins from distinct sequence families. Previously characterized gene Tip is accessory gene 1. See Supplementary Table 3 for more details on conserved gene functions.

phage or prophage (Supplementary Table S5). Although the observed diversity in carriage for these accessory genes is consistent with a role in modulating bacterial phenotypes as suggested previously (Cazares et al., 2014), no specific correlations could be discerned for gene complements that led to high resistance to further phage infection.

\section{Prophages provide phage resistance by a variety of} mechanisms

The diversity of resistance patterns and accessory gene content displayed by our lysogen collection suggested that a number of different mechanisms likely contributed to these effects. We first examined the contribution of phage repressor proteins to the resistance patterns. Prophages silence most phage genes through the activity of the repressor protein to maintain cell viability (Waldor and Friedman, 2005). By repressing genes essential for phage replication, phage repressors also mediate 'immunity' to subsequent infection by other phages that encode the same or a very similar repressor. To assess the contribution of phage repressors to the resistance patterns, we cloned and expressed seven different repressor genes from phages that mediated distinct patterns of resistance (Supplementary Figure S8a). Repressor activity accounted for only a small portion of the resistance exhibited by many lysogens (Supplementary Figure S8b, and separated horizontal groups on Figure 1). For example, expression of the repressor protein from phage JBD16C prevented plaquing of only itself and MP22, yet the JBD16C lysogen was resistant to 22 additional phages. Each of the repressors tested in our experiments displayed distinct and non-overlapping activities in mediating phage immunity, implying that each has a different DNA-binding specificity. This is supported by the diverse amino acids seen in the helix-turn-helix DNA-binding motifs (Heo et al., 2007; Stayrook et al., 2008) (Supplementary Figure S8a).

In the absence of extensive repressor-based immunity, other mechanisms must mediate the observed resistance profiles. The M2 group of phages (JBD44, JBD88b, JBD16, JBD13, JBD80) are dependent on the O-antigen (i.e. are inhibited by a $w b p L$ mutation) and not the T4P for infection of PA14. When present as a prophage, each of these five phages excludes the others from superinfection. However, this resistance is in part repressor-independent, as there are two different repressor proteins represented within this group (Figure 1 and Supplementary Figure S8). 
Outside this group, these five lysogens provide complete or partial resistance to four additional phages, none of which are blocked by either of the repressor proteins. These four additional phages require both the $\mathrm{O}$-antigen and the $\mathrm{T} 4 \mathrm{P}$ to infect PA14, and no phages that require the T4P alone are blocked by these lysogens. Interestingly, phages JBD5 and JBD93, which also depend on the O-antigen for infection, are not inhibited by this set of lysogens. These data suggested a modification to the O-antigen as the mechanism for resistance. As a lysogen of $P$. aeruginosa phage D3 had previously been shown to lead to serotype conversion (Newton et al., 2001), we determined the serotypes of these five lysogens using a slide agglutination test. However, the serotype of each of these lysogens was unchanged, showing that serotype conversion was not the reason for observed resistance. Thus, these prophages impart phage resistance to a subset of O-antigenrequiring phages, in a serotype-independent manner.

To investigate the mechanism of exclusion for T4P-dependent phages, we used a $\mathrm{K}^{+}$ion efflux assay to determine whether resistance manifests before or after genome injection. This experiment makes use of an ion-selective electrode to monitor the efflux of $\mathrm{K}^{+}$ions from bacterial cells during phage genome injection (Boulanger and Letellier, 1988,1992). Wildtype PA14 showed robust potassium efflux when challenged with several phages from our collection (Figure 4a; JBD26, JBD88a and JBD93). By contrast, the highly resistant PA14(JBD26) lysogen blocked $\mathrm{K}^{+}$ efflux when challenged with these same phages (Figure 4a), indicating that the phage resistance of this lysogen arose through changes at the cell surface or within the cell envelope, thereby preventing DNA injection. When the pilus-dependent phage JBD88a was used to challenge lysogens of JBD23 or JBD30, $\mathrm{K}^{+}$efflux levels similar those when infecting the nonlysogenic strain were observed, even though this phage cannot form plaques on either of these lysogens (Figure 4b). These data reveal that the block to replication of this phage occurs after DNA injection into these lysogens. As the plaquing of phage JBD88a is not inhibited by repressors that block the replication of phages JBD23 and JBD30, we conclude that the replication of JBD88a is blocked by a repressor-independent intracellular mechanism.

To determine if a phage that was inhibited in DNA injection as measured by the $\mathrm{K}^{+}$efflux assay was still able to bind to the cell surface, we assessed the ability of phage JBD24 to adsorb to the JBD26 lysogen (Supplementary Figure S9). When wild-type PA14 was challenged with phage JBD24, $80 \%$ of the phages bound to the bacteria within $8 \mathrm{~min}$. When the PA14(JBD26) lysogen was mixed with phage JBD24, no phage binding was detected in the same time period. This inhibition of binding was similar to that observed when phage JBD24 was incubated with a pilA mutant, which lacks the receptor needed for infection. This inhibition of phage binding to the bacterial cell is consistent with a modification to the bacterial cell surface by the PA14(JBD26) lysogen.

\section{Prophages can alter T4P function with no obvious} fitness cost

As the JBD26 prophage prevented phage adsorption, we hypothesized that this prophage and others in the same group might alter T4P function. $P$. aeruginosa strains use the T4P for twitching motility, whereby the bacteria move along a solid surface through a series of extensions and retractions of the T4P (Burrows, 2012). To assess T4P function in our collection of 30 lysogens, we measured twitching motility using a standard procedure that involves measuring the diameter of spreading of cells across a Petri plate (Burrows, 2012). JBD26 and eight other lysogens showed a $30-40 \%$ decrease in twitching motility diameter as compared with wild-type PA14 (Figure 5a), and in five lysogens (JBD24, JBD63b, JBD63c, JBD1 and JBD26) the morphology of the twitching zone was also altered (Figure 5b). These
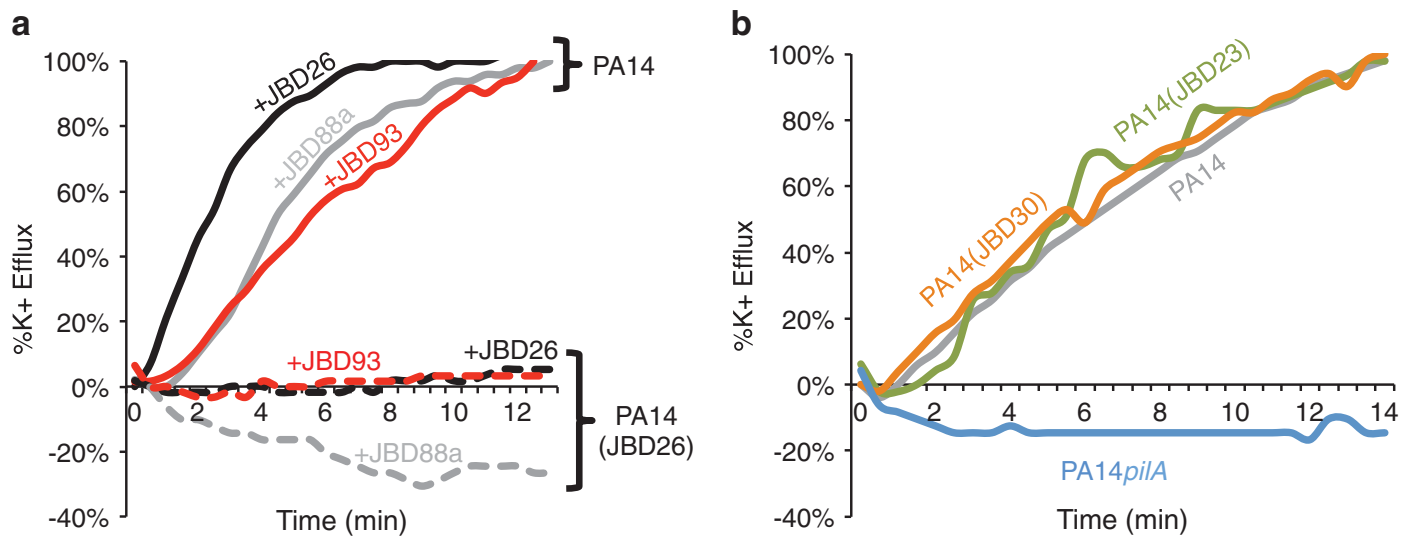

Figure 4 (a) PA14 or a PA14(JBD26) lysogen was infected with phage JBD26, JBD88a or JBD93 or (b) PA14 or PA14 lysogens of JBD23 or JBD30 were infected with phage JBD88a (at time 0) and the efflux of $\mathrm{K}^{+}$was measured over time. A PA14 $\Delta$ pilA mutant was also infected with JBD88a as a negative control. Efflux is represented as a percentage of the total $\mathrm{K}^{+}$efflux detected when wild-type PA14 was infected with a given phage (see Materials and methods). 

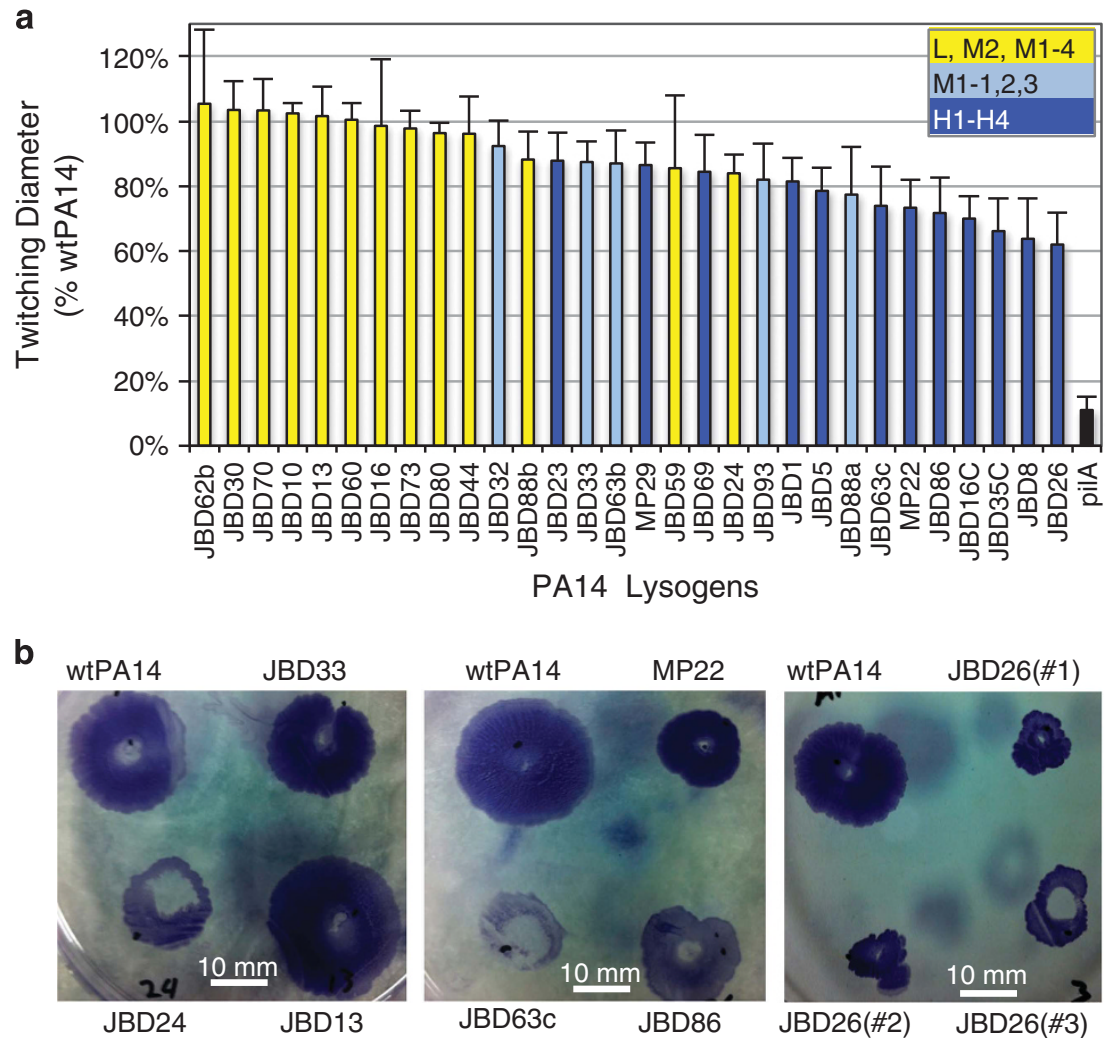

Figure 5 Prophages that impart strong phage resistance frequently alter T4P function. (a) The diameter of twitching motility zones for indicated PA14 lysogens is shown as a percentage, relative to wild-type PA14. Average value of three replicate experiments is shown and the error bars denote the standard deviations. (b) Representative images of twitching motility zones for PA14 lysogens visualized with crystal violet.

twitching defects were observed for the lysogens that displayed the strongest phage resistance patterns. For example, the integration of a JBD26 prophage decreased twitching motility by $40 \%$ compared with wild-type cells and provided resistance to all phages that rely on the $\mathrm{T} 4 \mathrm{P}$ for infection, including the previously characterized T4P-specific phages MP22 and D3112 (Heo et al., 2007). To determine if the observed twitching defect was due to a phageencoded gene or the interruption of a host gene due to integration of the prophage, we examined the twitching motility and integration sites of 10 individual JBD26 lysogens. Each lysogen showed identical phage resistance patterns (data not shown), had attenuated twitching motility (Figure 5b) and had unique integration sites in the host genome (Supplementary Figure S7b). Thus, the modification to the T4P that leads to the decreased twitching in this lysogen is most likely a result of the expression of one or more genes from the JBD26 prophage, and not from the disruption of a bacterial gene.

Cell surface modifications, in general, can be very costly for the host (Westra et al., 2015), especially if they interfere with important functions (Buckling and Brockhurst, 2012). The T4P is well known to be important for host infection (Feldman et al., 1998) and biofilm production (Feldman et al., 1998;
Jenkins et al., 2005). We therefore examined in a natural soil environment the fitness consequences of this T4P modification strategy by the JBD26 lysogen and compared it with the fitness cost associated with complete T4P loss. After 1 week, all strains reached similar densities growing in soil (Supplementary Figure S10). However, direct competition experiments between the strains revealed that fitness of the PA14(JBD26) strain was, within error, the same as the wild-type strain $(P=0.65)$, whereas fitness of a strain lacking the T4P was reduced by $\sim 60 \%$ compared with the wild-type strain $(P=0.0011)$ (Figure 6a). This shows that receptor modification by the prophage does not result in a detectable cost, consistent with the aligned fitness interests between the host and prophage. As the loss of the T4P from $P$. aeruginosa had previously been shown to mildly attenuate virulence in a Caenorhabditis elegans assay for bacterial pathogenesis (Tan et al., 1999), we also examined the ability of PA14(JBD26) to mediate slow killing in this model organism. Larval stage 4/young adult $C$. elegans were seeded on lawns of PA14 or PA14(JBD26), and the number of dead animals was recorded at multiple time points over $\sim 120 \mathrm{~h}$ (Figure 6b). The killing kinetics of the JBD26 lysogen was unchanged when compared with wildtype PA14. Thus, consistent with the evolutionary experiments in soil, we found no reduction in 


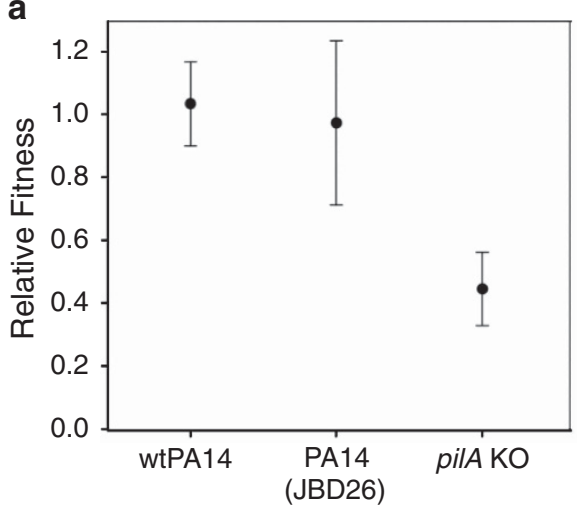

Strain vs. PA14::/acZ b

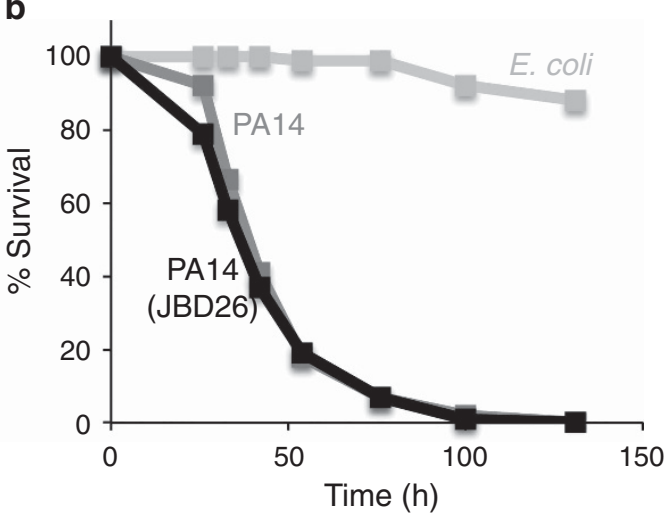

Figure 6 The JBD26 lysogen modifies pilus function with no discernable evolutionary cost. (a) PA14, PA14(JBD26) or a pilA mutant were competed with a lacZ-tagged PA14 strain in minimal media in autoclaved compost. The relative abundance of each strain was enumerated after 1 week of incubation. Error bars show the 95\% confidence interval. (b) 80-100 C. elegans nematodes were plated on lawns of $E$. coli OP50, wild-type PA14 or PA14(JBD26) and live and dead worms were scored over the course of $130 \mathrm{~h}$.

virulence associated with the increased phage resistance provided by JBD26 lysogen.

\section{Discussion}

The presence of prophages within bacterial genomes has been shown to markedly influence bacterial fitness and the movement of phages among bacterial strains has a significant role in the ongoing evolution of pathogens (Canchaya et al., 2003). As phage predation is a major threat to bacterial survival, there is strong selection for resistance to it. In this work, we systematically examined the ability of different prophages in a single $P$. aeruginosa strain background to resist superinfection. Diverse resistance patterns were observed, with some lysogens able to resist superinfection by $75 \%$ of the phages tested. The resistance was mediated through a variety of mechanisms, some of which affected the $\mathrm{T} 4 \mathrm{P}$ and $\mathrm{O}$-antigen, and others that functioned inside the cell. We found that 29 out of 30 prophages in our collection mediated significant resistance to at least three phages, and 12 lysogens mediated resistance to $>20$ different phages. These results demonstrate that almost every prophage has some effect on the ability of a lysogen to exclude non-self phages. Given that our phage collection was established from a highly diverse $P$. aeruginosa strain collection and that we tested every isolated phage that could form plaques on PA14, this result is likely to be representative of $P$. aeruginosa prophages in general.

Besides, the high frequency with which prophages exerted an effect on their host, another striking finding in this study was the wide diversity of both resistance phenotypes imparted by the prophages and plaquing behaviors of the phages tested. The lysogens displayed 12 distinct patterns of resistance to phages, and more than 10 distinct phage infectivity patterns can be seen (Figure 1).
Classic repressor-mediated immunity accounted for less than half of the phage resistance mediated by the lysogens. The high level of resistance displayed by the $\mathrm{H}$ group of lysogens was likely due to mechanisms related to the T4P as the phages inhibited by these lysogens required the T4P for infection. A previous study showed that the Tip protein from $P$. aeruginosa phage D3112 inhibits the function of the $\mathrm{T} 4 \mathrm{P}$ and prevents infection by phages requiring this structure (Chung et al, 2014). D3112 is closely related to the sequenced $\mathrm{T} 4 \mathrm{P}$-dependent phages described here, and four phages (MP29, JBD69, JBD23 and JBD26) encode identical or closely related homologs of Tip. Although the JBD26 lysogen showed greatly decreased twitching motility and was resistant to all pilus-requiring phages, lysogens of other Tip-encoding phages (JBD23, JBD69 and MP29) displayed twitching motilities equal to $80 \%$ of wild type and were only partially resistant to some T4P-dependent phages. In fact, while JBD23 encodes a Tip protein, potassium efflux experiments showed that the T4P-dependent phage JBD88a was still able to infect this lysogen. This may be due to variable expression levels of Tip from different lysogens. A number of prophages that conferred a high degree of phage resistance do not encode homologs of Tip, implying that there are other important resistance mechanisms in play. For example, while the M1-4 group of lysogens (JBD30, JBD59 and JBD60) was sensitive to many of the T4P-dependent phages, it was able to prevent infection by phage JBD88a. As potassium efflux experiments showed that the JBD88a genome was able to efficiently inject its genome into a JBD30 lysogen, the resistance mechanism must involve an intracellular mechanism that blocks replication at a subsequent step. In general, lysogens of T4P-dependent phages prevented infection by T4P-dependent phages and lysogens of $\mathrm{O}$-antigen-dependent phages prevented infection by the O-antigen-dependent phages. However, there 
were exceptions to this observation; phages JBD33 and JBD5, which could not form plaques on either pilus or O-antigen mutants, were not blocked by the O-antigen-dependent phage lysogens. Overall, these results highlight the complexity and variability of the interactions between this group of phages and their corresponding lysogens.

As alteration of T4P function appeared to be a frequently used means for prophages to inhibit phage replication, we examined this phenomenon more closely. Approximately one-third of the PA14 lysogens showed decreased twitching zones, and in several cases the morphology of the twitching zone was also altered, indicating some abrogation of $\mathrm{T} 4 \mathrm{P}$ function. Although several lysogens (e.g. JBD26, MP22 and JBD1) were as resistant to phages as the pilA mutant, which has no pili at all, these lysogens still showed twitching motility of $60-80 \%$ of wild type. This implied that the lysogens still had pili, but they were modified in some way to prevent phage adsorption. Remarkably, in contrast to the pilA mutant, the JBD26 prophage-mediated T4P alteration had no detectable evolutionary cost as shown by competition experiments in soil (Figure 6a) and killing kinetics in a C. elegans model system (Figure 6b). These data show that there is no detectable fitness trade-off associated with the phage resistance phenotype of the JBD26 lysogen; the lysogen is resistant to many different T4Pdependent phages without losing the important adaptive role of the T4P itself. This finding emphasizes a highly beneficial effect of this prophage on its host strain.

The variability of behaviors of the phages and prophages investigated here is surprising in light of the high degree of protein sequence similarity among many of the phages. Given the high sequence similarity among the conserved genes in these phage genomes, we hypothesize that the diverse lysogen properties and plaquing behaviors of these phages are the result of the variable accessory genes. Some of these genes are shared among many of these phages and others are unique to very few or even one phage. The prevalence and recognized importance of the highly variable 'accessory genome' in determining the phenotypic effects of phages and prophages confounds our ability to predict the impact of any given prophage. Although homologs of some of these accessory genes are widespread among diverse phages (Supplementary Table S5), the functions of these genes are mostly unknown. In previous studies, phage accessory genes have been shown to improve bacterial fitness by a variety of mechanisms, such as protecting against further phage invasion, increasing serum resistance and aiding host colonization (Bondy-Denomy and Davidson, 2014). This study represents the first systematic approach to address how a population of prophages influences phage resistance and bacterial behavior in $P$. aeruginosa, and it highlights the variability in the accessory genes even within a group of very closely related phage genomes. Further studies of the roles of phage accessory genes in increasing bacterial fitness are critical due to their impact on the evolution of bacterial populations, their roles in phage-phage competition, and their importance in infectious diseases.

\section{Materials and methods}

Strains and growth conditions

All strains and phages used in this study are listed in Supplementary Table S1 and Supplementary Table S2, respectively. All P. aeruginosa strains were grown on Lysogeny Broth (LB) agar or broth at $37^{\circ} \mathrm{C}$. Plaque assays were conducted at $30^{\circ} \mathrm{C}$ on LB agar plates with $0.7 \%$ LB top agar, both supplemented with $10 \mathrm{~mm} \mathrm{MgSO}_{4}$. For plaque assays, $150 \mu \mathrm{l}$ of overnight culture was mixed with $3 \mathrm{ml}$ of top agar, poured onto an LB agar plate and allowed to solidify, and then $4 \mu \mathrm{l}$ of serial dilutions of phage lysates were spotted on top and incubated overnight. Phage lysates were standardized to a titer in a range of $10^{7}-10^{8} \mathrm{PFU}$ per $\mathrm{ml}$ for the large-scale screen.

\section{Phage isolation and lysogen formation}

To induce prophages, strains were grown in LB at $37^{\circ} \mathrm{C}$ to an $\mathrm{OD}_{600}$ of 0.5 , and then mitomycin $\mathrm{C}$ was added to a final concentration of $3 \mu \mathrm{g} \mathrm{ml}^{-1}$. The cultures were incubated until lysis was visible (4-5 h after induction). A few drops of chloroform were added, and the cultures were incubated for $15 \mathrm{~min}$, and then centrifuged $10 \mathrm{~min}$ at $10000 \mathrm{~g}$ to remove bacterial cell debris. A few drops of chloroform were added to the cleared supernatant and the sterile lysate was stored at $4{ }^{\circ} \mathrm{C}$. Ten-fold serial dilutions of the lysates were applied to lawns of bacterial indicator strains to identify permissive bacterial hosts.

Phage isolation was performed by mixing permissive cells with phages in top agar and plating the mixture on LB plates to isolate single plaques. Individual plaques were picked and resuspended in $\mathrm{SM}$ buffer (100 mM NaCl, $8 \mathrm{~mm} \mathrm{MgSO}_{4}, 50 \mathrm{~mm}$ Tris-HCl ( $\mathrm{pH} 7.5), 0.01 \%$ gelatin). Each phage was plaque-purified three times before use. High titer phage stocks were prepared by soaking plates with near-confluent phage lysis with $4 \mathrm{ml}$ of SM buffer for 30 min with subsequent collection of the buffer. For further concentration and purification, phages were precipitated with polyethylene glycol $8000(10 \%$ $\left(\mathrm{w} \mathrm{v}^{-1}\right)$ ), followed by two rounds of cesium chloride equilibrium centrifugation. The resulting phage suspension was dialyzed in SM buffer, which was then used for downstream applications such as DNA extraction and electron microscopy.

Lysogens were created by plating serial dilutions of a purified phage on PA14 and streak platingresistant bacteria from the inside of a clearing to single colonies on LB agar. After confirming that individual colonies were resistant to the challenge 
phage, putative lysogens were grown in LB to $\mathrm{OD}_{600}=0.5$, mitomycin $\mathrm{C}$ induced and the resulting lysates were plated on PA14 to confirm that the original infecting phage was produced.

\section{Bioinformatics of phage genomes}

To analyze the proteomes of the T4P-dependent (MP22-like) phages, all proteins encoded by these phages were clustered using CD-HIT (Huang et al., 2010) using a $40 \%$ amino-acid sequence identity cutoff. Homologs of proteins designated as 'conserved proteins' were encoded in all 12 of the MP22like phage genomes. In 31 out $44(70 \%)$ conserved protein families, the average pairwise aminoacid sequence identity among the 12 homologs was $>85 \%$, and often exceeded $90 \%$ (see Supplementary Table S4). In the other families, the proteins clearly clustered into two or more groups, but these groups were clearly related ( $>30 \%$ sequence identity). The pairwise sequence identity within subclusters was always $>85 \%$. Functions for conserved proteins were assigned using PSI-BLAST searches to detect protein sequence similarity with homologs of known function. HHpred was used to detect distant similarities between proteins and sequence families or protein structures of known function. BLAST searches were performed against a database of Refseq complete bacterial and phage genomes that contained 755 tailed phage genomes and 2119 bacterial genomes (downloaded in April 2013). We have used this smaller database because we have annotated most of the phages and prophages in this database (manuscript to be submitted).

\section{Motility assays}

Twitching motility was assessed as follows: a single colony was stabbed through a $1 \%$ LB agar plate and the plates were inverted and incubated for $48 \mathrm{~h}$ at $37^{\circ} \mathrm{C}$. The agar was carefully removed and cells adhered to the Petri plate were stained with $1 \%$ $\left(\mathrm{w} \mathrm{v}^{-1}\right)$ crystal violet for $1 \mathrm{~min}$, and then washed three times with distilled water. The diameter of the twitch zone was measured for each of the mutants and compared with wild-type PA14.

\section{Electron microscopy}

Cesium chloride-purified phage stocks were examined with transmission electron microscopy after negative staining with $2 \%\left(\mathrm{w} \mathrm{v}^{-1}\right)$ uranyl acetate as described previously (Pell et al, 2009). Images were taken with a Hitachi H-7000 microscope at magnification levels of $\times 70000-200000$.

\section{Potassium efflux assays}

Bacteria were grown in $\mathrm{LB}$ to $\mathrm{OD}_{600}=0.5$, at which point they were collected by centrifugation, washed two times in SM buffer and resuspended in $5 \mathrm{ml}$ of $\mathrm{SM}$. The cell suspension was brought to $37^{\circ} \mathrm{C}$, the potassium-selective electrode was put in the sample and the reading was allowed to stabilize for 3-5 min. Cesium chloride-purified phage in SM buffer were added at a multiplicity of infection of $\sim 100$, the cells and phage were mixed thoroughly and $\mathrm{mV}$ readings were recorded every $5 \mathrm{~s}$ for $20 \mathrm{~min}$. The data shown in Figure 4 were normalized to time 0 when the phage was added to the cell suspension. Upon infection with a given phage, the maximum efflux value was determined for wild-type PA14 at the end point where the signal stabilized following phage addition. This value was set at $100 \%$ and used to normalize to the experiments with the same phage on other hosts (i.e. lysogens and pilA mutant).

\section{Competition experiments}

The following strains were inoculated into $10 \mathrm{ml} \mathrm{LB}$ and incubated at $37^{\circ} \mathrm{C}$ while shaking at 180 r.p.m.: $P$. aeruginosa strain PA14, $P$. aeruginosa csy3::lacZ (referred to as LacZ), P. aeruginosa strain PA14 carrying prophage JBD26 (referred to as WT(JBD26)) and $P$. aeruginosa Tn::pilA (referred to as pilA KO) (Liberati et al., 2006). After overnight growth, cultures were centrifuged at 3500 r.p.m. for $15 \mathrm{~min}$ and the pellets were resuspended in $10 \mathrm{ml} 1 \times \mathrm{M} 9$ salts. For every competition experiment, $20 \mathrm{ml}$ of $1 \mathrm{x}$ M9 salts was mixed with $100 \mu$ l of each strain. The resulting mixture was applied to $20 \mathrm{~g}$ of double autoclaved multipurpose compost contained in a square polypropylene plate and incubated at $26^{\circ} \mathrm{C}$ with $70 \%$ humidity. Samples were taken at $t=0$ (immediately after mixing the strains) and after 1 week. Cells were serially diluted in M9 salts and plated on LB agar supplemented with $50 \mu \mathrm{g} \mathrm{ml}^{-1}$ X-gal (5-bromo-4-chloro-3-indolyl- $\beta$-D-galactopyranoside). After overnight incubation, the relative frequency of each strain was determined by counting blue and white colonies. Each competition experiment was performed in six replicates. For statistical analyses, relative frequencies of each strain at $t=0$ and $t=1$ week was used to calculate the relative fitness (rel. fitness $=(($ fraction strain $\mathrm{A}$ at $t=1$ week $) \times(1-($ fraction stain A at $t=0))) /(($ fraction strain A at $t=0) \times(1-($ fraction strain $\mathrm{A}$ at $t=1$ week $))$ ), which were used for Student's T-test. The statistical analyses were carried out using the JMP (v.10, Buckinghamshire, UK) software. PCR was used to confirm that lysogenic strains maintained the prophage (12 clones per replicate).

\section{Southern blot}

Genomic DNA was purified from PA14 or indicated lysogens, digested with $\mathrm{NcoI}$ and separated by gel electrophoresis on a $0.8 \%$ TAE-agarose gel. The gel was washed in $0.25 \mathrm{M} \mathrm{HCl}$ for $10 \mathrm{~min}$, rinsed in water and washed in $0.4 \mathrm{~N} \mathrm{NaOH} / 0.6 \mathrm{M} \mathrm{NaCl}$ for 30 min. The DNA was transferred to a GeneScreen plus nylon membrane with a vacuum blotter for 60-120 min while adding a $20 \times$ saline-sodium 
citrate (SSC) solution. Finally, the nylon membrane was washed in blocking buffer $(50 \%$ formamide, $5 \times$ Denhardts solution, $0.5 \%$ sodium dodecyl sulfate (SDS), $6 \times$ SSC and $100 \mu \mathrm{gl}^{-1}$ herring sperm DNA) at $42{ }^{\circ} \mathrm{C}$ for $2 \mathrm{~h}$. The blocking buffer was then replaced with a fresh solution of the same buffer but with herring sperm DNA omitted and $5 \times 10^{6}$ counts per minute of ${ }^{32} \mathrm{P}$-labeled phage DNA added. The probe was generated from a random hexamer extension of purified phage genomic DNA. After probing overnight at $42{ }^{\circ} \mathrm{C}$, the membrane was washed two times for $10 \mathrm{~min}$ at room temperature $(2 \times$ SSC, $1 \%$ SDS $)$, two times for $30 \mathrm{~min}$ at $65^{\circ} \mathrm{C}(2 \times \mathrm{SSC}, 1 \%$ SDS $)$ and once for $10 \mathrm{~min}$ at room temperature (0.2 $\times$ SSC, $0.1 \%$ SDS). The membrane was wrapped in Saran Wrap, exposed to a phosphoscreen and developed on a Typhoon imager (GE Healthcare, Mississauga, ON, Canada).

\section{Caenorhabditis elegans virulence assay}

This protocol was followed directly from Tan et al. (1999). Indicated PA14-derived lysogen overnight cultures were plated on slow killing medium and incubated for $24 \mathrm{~h}$ at $37^{\circ} \mathrm{C}$ and room temperature for $24 \mathrm{~h}$. Approximately $40 \mathrm{~L} 4 /$ young adult stage worms were transferred to the $P$. aeruginosa plates in duplicate. Live and dead worms were scored manually over the time course of the experiment. Dead worms were removed from the plates to ensure accurate counting.

\section{Adsorption assay}

Cells were grown for $3 \mathrm{~h}$ in $\mathrm{LB}+10 \mathrm{~mm} \mathrm{MgSO}_{4}$ to an $\mathrm{OD}_{600}=0.5$. Phages were added at a multiplicity of infection $=0.1$ and incubated for 0 or $8 \mathrm{~min}$. At those time points, an aliquot was removed from the sample and diluted in cold buffer containing chloroform. This mixture was centrifuged to pellet cells and attached phages. The supernatant was titrated on a sensitive strain to quantify the unattached phage.

Phage genome sequencing and analysis

Purified phages were isolated in a cesium chloride gradient and treated with $20 \mathrm{mM}$ EDTA, $50 \mu \mathrm{g} \mathrm{ml}^{-1}$ proteinase $\mathrm{K}$ and $0.5 \%$ SDS for $1 \mathrm{~h}$ at $56^{\circ} \mathrm{C}$. The genomic DNA was isolated by phenol-chloroform extraction and precipitated with $0.3 \mathrm{M}$ sodium acetate $(\mathrm{pH}$ 7.0) and two volumes of ice-cold 100\% ethanol. The DNA was collected by centrifugation at 10000 r.p.m. for $2 \mathrm{~min}$, the pellet was washed with $70 \%$ ethanol and then air-dried. The DNA pellet was resuspended in water and frozen at $-20^{\circ} \mathrm{C}$.

Phage genome sequencing was conducted on the Illumina platform (Illumina GAIIx, San Diego, CA, USA) with paired-end reads from a multiplexed run, sequencing 12 phage genomes per lane. Assembly was run on Velvet and coverage was $>\times 400$ for each phage. Genome analysis was conducted primarily with BLASTn, BLASTp (Altschul et al., 1990) and
RAST (Aziz et al., 2008) programs. Putative gene functions were assigned through BLAST searches and comparisons using HHpred (Söding et al., 2005) and HMM-HMM comparison was used for protein homology detection (Söding, 2005).

\section{Phylogenetic analysis of $P$. aeruginosa isolates}

Seven genes were used for the $P$. aeruginosa multilocus sequence typing, as per pubMLST (http:// pubmlst.org/paeruginosa/info/primers.shtml). Of the 87 isolates used to build the tree shown in Supplementary Figure S1, complete sequences for all 7 genes were used for 73 isolates, whereas 13 isolates were aligned using 6/7 complete gene sequences with 1 incomplete sequence, and 1 isolate was aligned using 5/7 complete sequences and 2 incomplete sequences. The sequence alignment (by ClustalW) and phylogenetic analysis were both carried out in MEGA5 (Tamura et al., 2011).

The evolutionary history was inferred by using the maximum-likelihood method based on the TamuraNei model (Tamura and Nei, 1993). The tree with the highest log likelihood (-3900.3704) was used. Initial tree(s) for the heuristic search were obtained automatically by applying neighbor-joining and BioNJ algorithms to a matrix of pairwise distances estimated using the maximum composite-likelihood approach, and then selecting the topology with superior log-likelihood value. Codon positions

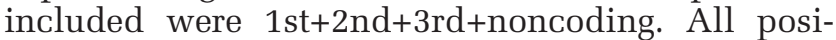
tions containing gaps and missing data were eliminated for a total of 1701 positions in the final data set.

\section{Conflict of Interest}

The authors declare no conflict of interest.

\section{Acknowledgements}

This work was supported by operating grants from the Canadian Institutes of Health Research to KLM (MOP-136845) and ARD (XNE-86943). JB-D was supported by a CIHR Canada Graduate Scholarship Doctoral Award and an Ontario Graduate Scholarship. DSG is supported by NSERC. ERW was supported by NERC and Wellcome Trust. AB acknowledges NERC, AXA, BBSRC and the Royal Society for funding. We thank Andrew Spence, Kathy Shire, Steven Doyle and Battista Calvieri for advice and technical assistance. We also thank Derrick Fouts at the J Craig Venter Institute for phage sequencing (NIH U54 AI84844-01).

\section{References}

Ackermann H-W, Cartier C, Slopek S, Vieu JF. (1988). Morphology of Pseudomonas aeruginosa typing 
phages of the Lindberg set. Ann Inst Pasteur Virol 139: 389-404.

Altschul SF, Gish W, Miller W, Myers EW, Lipman DJ. (1990). Basic local alignment search tool. J Mol Biol 215: 403-410.

Aziz RK, Bartels D, Best AA, DeJongh M, Disz T, Edwards RA et al. (2008). The RAST Server: rapid annotations using subsystems technology. BMC genomics 9: 75-99.

Bergh O, Børsheim KY, Bratbak G, Heldal M. (1989). High abundance of viruses found in aquatic environments. Nature 340: 467-468.

Bondy-Denomy J, Davidson AR. (2014). When a virus is not a parasite: the beneficial effects of prophages on bacterial fitness. J Microbiol (Seoul, Korea) 52: 235-242.

Bondy-Denomy J, Pawluk A, Maxwell KL, Davidson AR. (2013). Bacteriophage genes that inactivate the CRISPR/Cas bacterial immune system. Nature 493: 429-432.

Boulanger P, Letellier L. (1988). Characterization of ion channels involved in the penetration of phage T4 DNA into Escherichia coli cells. $J$ Biol Chem 263: 9767-9775.

Boulanger P, Letellier L. (1992). Ion channels are likely to be involved in the two steps of phage T5 DNA penetration into Escherichia coli cells. J Biol Chem 267: 3168-3172.

Bourkal'tseva MV, Krylov SV, Kropinski AM, Pleteneva EA, Shaburova OV, Krylov VN. (2011). Bacteriophage phi297, a new species of Pseudomonas aeruginosa temperate phages with a mosaic genome: potential use in phage therapy. Russ J Genet 47: 794-798.

Bradley DE. (1972). Evidence for the retraction of Pseudomonas aeruginosa RNA phage pili. Biochem Biophys Res Commun 47: 142-149.

Bradley DE. (1973). Basic characterization of a Pseudomonas aeruginosa pilus-dependent bacteriophage with a long noncontractile tail. J Virol 12: 1139-1148.

Buckling A, Brockhurst M. (2012). Bacteria-virus coevolution. Adv Exp Med Biol 751: 347-370.

Burrows LL. (2012). Pseudomonas aeruginosa twitching motility: type IV pili in action. Annu Rev Microbiol 66: 493-520.

Canchaya C, Proux C, Fournous G, Bruttin A, Brüssow H. (2003). Prophage genomics. Microbiol Mol Biol Rev 67: 238-276.

Cazares A, Mendoza-Hernández G, Guarneros G. (2014). Core and accessory genome architecture in a group of Pseudomonas aeruginosa Mu-like phages. BMC Genomics 15: 1146.

Chung I-Y, Cho Y-H. (2012). Complete genome sequences of two Pseudomonas aeruginosa temperate phages, MP29 and MP42, which lack the phage-host CRISPR interaction. I Virol 86: 8336.

Chung I-Y, Jang HJ, Bae HW, Cho Y-H. (2014). A phage protein that inhibits the bacterial ATPase required for type IV pilus assembly. Proc Natl Acad Sci USA 111: 11503-11508.

Cumby N, Edwards AM, Davidson AR, Maxwell KL. (2012). The bacteriophage HK97 gp15 moron element encodes a novel superinfection exclusion protein. J Bacteriol 194: 5012-5019.

Feldman M, Bryan R, Rajan S, Scheffler L, Brunnert S, Tang $\mathrm{H}$ et al. (1998). Role of flagella in pathogenesis of Pseudomonas aeruginosa pulmonary infection. Infect Immun 66: 43-51.
Heo Y-J, Chung I-Y, Choi KB, Lau GW, Cho Y-H. (2007). Genome sequence comparison and superinfection between two related Pseudomonas aeruginosa phages, D3112 and MP22. Microbiology (Reading, England) 153(Part 9): 2885-2895.

Huang Y, Niu B, Gao Y, Fu L, Li W. (2010). CD-HIT Suite: a web server for clustering and comparing biological sequences. Bioinformatics 26: 680-682.

James CE, Fothergill JL, Kalwij H, Hall AJ, Cottell J, Brockhurst MA et al. (2012). Differential infection properties of three inducible prophages from an epidemic strain of Pseudomonas aeruginosa. BMC Microbiol 12: 216.

Jenkins ATA, Buckling A, McGhee M, ffrench-Constant RH. (2005). Surface plasmon resonance shows that type IV pili are important in surface attachment by Pseudomonas aeruginosa. JR Soc Interface 2: 255-259.

Kropinski AM. (2000). Sequence of the genome of the temperate, serotype-converting Pseudomonas aeruginosa bacteriophage D3. J Bacteriol 182: 6066-6074.

Liberati NT, Urbach JM, Miyata S, Lee DG, Drenkard E, Wu G et al. (2006). An ordered, nonredundant library of Pseudomonas aeruginosa strain PA14 transposon insertion mutants. Proc Natl Acad Sci USA 103: 2833-2838.

Newton GJ, Daniels C, Burrows LL, Kropinski AM, Clarke AJ, Lam JS. (2001). Three-component-mediated serotype conversion in Pseudomonas aeruginosa by bacteriophage D3. Mol Microbiol 39: 1237-1247.

Parkins MD, Glezerson BA, Sibley CD, Sibley KA, Duong J, Purighalla S et al. (2014). Twenty-five-year outbreak of Pseudomonas aeruginosa infecting individuals with cystic fibrosis: identification of the prairie epidemic strain. J Clin Microbiol 52: 1127-1135.

Pawluk A, Bondy-Denomy J, Cheung VHW, Maxwell KL, Davidson AR. (2014). A new group of phage antiCRISPR genes inhibits the type I-E CRISPR-Cas system of Pseudomonas aeruginosa. mBio 5: e00896.

Pell LG, Kanelis V, Donaldson LW, Howell PL, Davidson AR. (2009). The phage lambda major tail protein structure reveals a common evolution for long-tailed phages and the type VI bacterial secretion system. Proc Natl Acad Sci USA 106: 4160-4165.

Salmon KA, Freedman O, Ritchings BW, DuBow MS. (2000). Characterization of the lysogenic repressor (c) gene of the Pseudomonas aeruginosa transposable bacteriophage D3112. Virology 272: 85-97.

Samson JE, Magadán AH, Sabri M, Moineau S. (2013). Revenge of the phages: defeating bacterial defences. Nat Rev Microbiol 11: 675-687.

Söding J. (2005). Protein homology detection by HMMHMM comparison. Bioinformatics (Oxford, England) 21: 951-960.

Söding J, Biegert A, Lupas AN. (2005). The HHpred interactive server for protein homology detection and structure prediction. Nucleic Acids Res 33: W244-W248.

Stayrook S, Jaru-Ampornpan P, Ni J, Hochschild A, Lewis M. (2008). Crystal structure of the lambda repressor and a model for pairwise cooperative operator binding. Nature 452: 1022-1025.

Sun X, Göhler A, Heller KJ, Neve H. (2006). The ltp gene of temperate Streptococcus thermophilus phage TP-J34 confers superinfection exclusion to Streptococcus thermophilus and Lactococcus lactis. Virology 350: 146-157. 
Susskind MM, Wright A, Botstein D. (1974). Superinfection exclusion by $\mathrm{P} 22$ prophage in lysogens of Salmonella typhimurium. IV. Genetics and physiology of sieB exclusion. Virology 2: 367-384.

Suttle CA. (2005). Viruses in the sea. Nature 437: 356-361.

Suttle CA. (2007). Marine viruses-major players in the global ecosystem. Nat Rev Microbiol 5: 801-812.

Tamura K, Nei M. (1993). Estimation of the number of nucleotide substitutions in the control region of mitochondrial DNA in humans and chimpanzees. Mol Biol Evol 10: 512-526.

Tamura K, Peterson D, Peterson N, Stecher G, Nei M, Kumar S. (2011). MEGA5: molecular evolutionary genetics analysis using maximum likelihood, evolutionary distance, and maximum parsimony methods. Mol Biol Evol 28: 2731-2739.

Tan MW, Rahme LG, Sternberg JA, Tompkins RG, Ausubel FM. (1999). Pseudomonas aeruginosa killing of Caenorhabditis elegans used to identify $P$. aeruginosa virulence factors. Proc Natl Acad Sci USA 96: 2408-2413.

Uc-Mass A, Loeza EJ, de la Garza M, Guarneros G, Hernández-Sánchez J, Kameyama L. (2004). An orthologue of the cor gene is involved in the exclusion of temperate lambdoid phages. Evidence that Cor inactivates FhuA receptor functions. Virology 329: 425-433.
Waldor MK, Friedman DI. (2005). Phage regulatory circuits and virulence gene expression. Curr Opin Microbiol 8: 459-465.

Wang PW, Chu L, Guttman DS. (2004). Complete sequence and evolutionary genomic analysis of the Pseudomonas aeruginosa transposable bacteriophage D3112. J Bacteriol 186: $400-410$.

Westra ER, van Houte S, Oyesiku-Blakemore S, Makin B, Broniewski JM, Best A et al. (2015). Parasite exposure drives selective evolution of constitutive versus inducible defense. Curr Biol 25: 1043-1049.

Winstanley C, Langille MGI, Fothergill JL, Kukavica-Ibrulj I, Paradis-Bleau C, Sanschagrin F et al. (2009). Newly introduced genomic prophage islands are critical determinants of in vivo competitiveness in the Liverpool Epidemic Strain of Pseudomonas aeruginosa. Genome Res 19: 12-23.

Wommack KE, Colwell RR. (2000). Virioplankton: viruses in aquatic ecosystems. Microbiol Mol Biol Rev 64: 69-114.

Zegans ME, Wagner JC, Cady KC, Murphy DM, Hammond JH, O'Toole GA. (2009). Interaction between bacteriophage DMS3 and host CRISPR region inhibits group behaviors of Pseudomonas aeruginosa. J Bacteriol 191: $210-219$.

Supplementary Information accompanies this paper on The ISME Journal website (http://www.nature.com/ismej) 